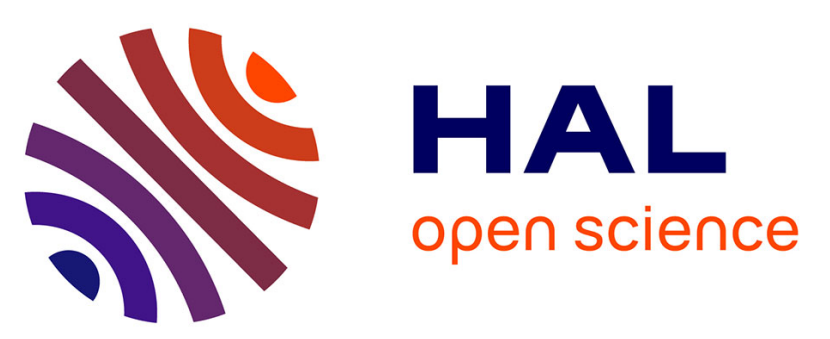

\title{
Monte Carlo Simulation-Based Probabilistic Assessment of DG Penetration in Medium Voltage Distribution Networks
}

\author{
Enrico Zio, Maurizio Delfanti, Livio Giorgio, Valeria Olivieri, Giovanni \\ Sansavini
}

\section{To cite this version:}

Enrico Zio, Maurizio Delfanti, Livio Giorgio, Valeria Olivieri, Giovanni Sansavini. Monte Carlo Simulation-Based Probabilistic Assessment of DG Penetration in Medium Voltage Distribution Networks. International Journal of Electrical Power \& Energy Systems, 2015, 64, pp.852-860. 10.1016/j.ijepes.2014.08.004 . hal-01259656

\section{HAL Id: hal-01259656 \\ https://hal.inria.fr/hal-01259656}

Submitted on 20 Jan 2016

HAL is a multi-disciplinary open access archive for the deposit and dissemination of scientific research documents, whether they are published or not. The documents may come from teaching and research institutions in France or abroad, or from public or private research centers.
L'archive ouverte pluridisciplinaire HAL, est destinée au dépôt et à la diffusion de documents scientifiques de niveau recherche, publiés ou non, émanant des établissements d'enseignement et de recherche français ou étrangers, des laboratoires publics ou privés. 


\title{
Monte Carlo Simulation-Based Probabilistic Assessment of DG Penetration in Medium Voltage Distribution Networks
}

\author{
E. Zio, M. Delfanti, L. Giorgi, V. Olivieri, G. Sansavini
}

\begin{abstract}
With the growing use of renewable energy sources, Distributed Generation (DG) systems are rapidly spreading. Embedding DG to the distribution network may be costly due to the grid reinforcements and control adjustments required in order to maintain the electrical network reliability. Deterministic load flow calculations are usually employed to assess the allowed DG penetration in a distribution network in order to ensure that current or voltage limits are not exceeded. However, these calculations may overlook the risk of limit violations due to uncertainties in the operating conditions of the networks. To overcome this limitation, related to both injection and demand profiles, the present paper addresses the problem of DG penetration with a Monte Carlo technique that accounts for the intrinsic variability of electric power consumption. The power absorbed by each load of a Medium Voltage network is characterized by a load variation curve; a probabilistic load flow is then used for computing the maximum DG power that can be connected to each bus without determining a violation of electric constraints. A distribution network is studied and a comparison is provided between the results of the deterministic load flow and probabilistic load flow analyses.
\end{abstract}

Keywords-Distributed Generation; Electrical Distribution Network; Probabilistic Load Flow; Monte Carlo Simulation

\section{INTRODUCTION}

It is becoming increasingly difficult to satisfy the ever growing energy need of modern societies, due to the instability of the prices of the energy sources and the concerns to reduce greenhouse gas emissions (e.g. 2020-20 targets [1]). In this scenario, the electric infrastructure witnesses a major change: electricity, which is traditionally generated in large, centralized plants connected to the transmission network, is increasingly produced in small or medium units connected to the distribution networks, possibly close to the end users [2]. This innovative paradigm is called Distributed Generation (DG), which is defined as small/medium scale electric power generation within distribution networks (or on the customer side of the network) based on Renewable Energy Sources (RES) and Combined Heat and Power (CHP) technologies. Generally, DG technologies lead to emissions of greenhouse gases significantly lower than fossil fuel-based technologies, which is the major environmental concern nowadays. DG reduces in most cases the amount of energy lost in transmitting electricity, and allows more flexibility in terms of primary sources that are converted into electric energy [3]. In addition, DG allows a deeper utilization and integration of RES [4], [5].

DG is running particularly fast in Europe, where significant incentive schemes and other benefits such as

E. Zio is with the Ecole Centrale Paris and Supelec, Paris, France. He is also with the Department of Energy, Politecnico di Milano, Milan, Italy (e-mail: enrico.zio@ecp.fr; enrico.zio@supelec.fr; enrico.zio@polimi.it).

M. Delfanti is with the Department of Energy, Politecnico di Milano, Milan, Italy (e-mail: maurizio.delfanti@polimi.it).

L. Giorgi is with the Department of Energy, Politecnico di Milano, Milan, Italy (e-mail: livio.giorgi@ @ail.polimi.it).

V. Olivieri is with the Department of Energy, Politecnico di Milano, Milan, Italy (e-mail: valeria.oliveiri@ mail.polimi.it).

G. Sansavini is with the Institute of Energy Technology, ETH Zurich, Switzerland (e-mail: sansavig@ethz.ch). This work was performed when the author was with Department of Energy, Politecnico di Milano, Milan, Italy. 
simplified access to the grid and priority in dispatching have been promoted by many Member States in order to match the targets decided by the European institutions. As a consequence of the important share of RES already connected, especially to Low Voltage (LV) and Medium Voltage (MV) networks, new technical challenges have to be faced both at the transmission network level and at the distribution network level. As for transmission networks, it can be assumed that this system has a quite advanced structure and does not require substantial technological changes in order to face the DG increase [6]: the most important issues are related to the unpredictability of RES, that has to be faced by proper countermeasures (e.g. increased spinning reserve margins and/or storage systems). On the contrary, distribution networks (MVLV) are traditionally operated radially (i.e., energy flows mainly from the primary substation to the lower voltage levels) and without an on-line control of voltages and currents in the DG Point of Common Coupling (PCC). The Distribution System Operators (DSOs) generally follow the "fit and forget approach", that results in over-sizing the distribution grid in order to avoid the infringement of technical constraints during the few periods of high DG injection (strong wind or sunshine) and of low load absorption. Such architecture, chosen when DG was very rare, today, in presence of very high levels of DG penetration, can be considerably impacted for operation, control, protection and reliability issues [7], [8], [9], as DG involves a complete redeployment of both active and reactive power flows along distribution networks. In order to cope with this situation, appropriate Load Flow (LF) calculations are needed to assess the allowed DG penetration level for a given network, in terms of active DG power that can be connected to a distribution network in accordance with technical constraints (and, obviously, with no modification in structures and no deterioration in reliability and quality of service). However, a realistic evaluation of where and when a constraint infringement occurs can be difficult to obtain by simply using traditional Deterministic LF analysis (DLF), [10]-[14], that neglects the uncertainties of many parameters affecting power system operation.

The vast majority of current methods for DG sizing and placement for the planning of distribution networks overlooks or partially accounts for uncertainty representation and propagation. Optimization with respect to diverse DG issues, e.g. number and capacity of DG units, location, type, and impact on power system operating characteristics (electric losses, costs, voltage profile, emissions stability and reliability), is the most investigated method for DG penetration assessment in the literature. In [12], an OPF method for assessing the DG capacity of network is proposed which for the first time includes voltage step limits (rapid voltage changes) on loss of a generator, in addition to voltage level and thermal constraints. The potential for DG connection to a selected number of buses is assessed and the optimization problem is solved by a general reduced gradient solver. A mixed integer non-linear programming approach for determining optimal location and number of distributed generators is presented in [15], which is based on the minimization of total fuel costs and line losses. The mixed integer non-linear programming problem is solved using a discrete continuous optimization package solver. The active and reactive power demand at each bus is based on peak load and does not consider multiple load conditions. A planning and production simulation model for optimizing DG in the form of mixed integer linear stochastic programming is developed in [16] and solved using generalized Benders decomposition. The model accounts for random load and generator availability variations without explicitly considering every possible scenario by resorting to an approximation procedure which clusters hourly loads into groups represented by the average load of the group. Simplifying linearized power flow [16] and constant power factors [17] are adopted in models which consider multiple load conditions across time for network planning. Optimization problems concerning the size and location of multiple DGs are also solved through metaheuristics. The minimization of power loss with voltage regulation as a constraint is achieved using hybrid particle swarm optimization (PSO) and a natural selection mechanism in [18]. The effect of load growth on different parameters of the power system is considered for a time horizon of 5 years, but the impact of uncertainties on these estimates is not accounted for. Quadratic programming is combined with PSO in [19] for the optimization of DG capacity and operational strategy for energy management based on investment, operational and maintenance costs as well as revenues including those from reduction of pollution. Uncertainties associated with electrical power price are represented and propagated through the fuzzy set theory. Genetic algorithms 
(GA) are used in [20] to minimize electric losses and guarantee acceptable reliability level and voltage profiles for the optimization of the allocation and sizing of DG units. In the adopted problem formulation, it is considered that the load demand is constant during the planning period. Time variant loads are modeled in [21] considering five different load values in a method for optimally allocating DG for profit maximization, reduction of losses and improvement in voltage regulation. A GA-based method is developed to obtain optimal position and optimal size of single and multiple DG resources. Three different loading levels are considered in [22], where DG is considered for multistage distribution system expansion planning. A combined GA and optimal power flow is developed to minimize investment, operation, and outage costs of the system. In [23], mixed-integer programming methods and GA methods are compared with respect to the solution to the problem of optimal siting and sizing of DGs. The issues of considering multiple load conditions across time and probabilistic load profiles have been set aside in order to address strong combinatorial aspects in a timely manner.

The hosting-capacity method is also suggested in the literature to evaluate the capability of medium voltage buses to accept power injection from DGs [24]. This approach is based on the fact that every change in the generation or in the load impacts the performance of the power system. Therefore, the impact of DG can be quantified only by using a set of performance indicators. The hosting capacity is defined as the amount of DG for which the performance becomes unacceptable. An algorithm considering the degree of DG that can be accepted without endangering the reliability or quality of power with respect to two limits, i.e. overvoltage and overcurrent, is developed in [11]. Three kinds of limits are adopted in [14]: thermal limits, i.e. current limit of each branch, voltage limits (i.e., supply voltage variations) for each bus, and rapid voltage changes associated to sudden variations (e.g. trip) of generator power output. The hosting capacity is usually evaluated for each hour during the planning horizon for DG but the possible effects that uncertainty may have on the likelihood of limit violation are not quantified.

The adequacy assessment of DG on power system is often based on Monte Carlo simulations that consider the random parameters of the DG. For example, Monte Carlo simulation is exploited for optimal DG allocation and sizing in distribution systems considering multi-level load models, in order to minimize the costs of active and reactive losses and improve the voltage profile and reliability [25]; or to assess the impact of DG connected to distribution networks on investment deferral in the long-term [26] or on voltage and current limits [27], [28] according to the different DG penetration and concentration levels, and to DG technology mix. The adequacy of distribution system with consideration of RES injections is assessed using the inherent variability of primary energy [29], that is important also to find the proportion of the RES for maximum generating capacity or lowest generation cost subject to lowest emission or to lowest capital cost [30], and to estimate the average amount of unsupplied loads [31]. Monte Carlo simulations are also carried out in order to find the optimal placement of DG resources in power systems [32]-[36].

In our work, the focus is on load modeling. According to a practical approach, we observe that, in most cases (according to the regulations in place in many EU countries, and especially in Italy) DSOs are not responsible for optimizing size and location of DG units to be connected to the distribution network; on the other hand, they are fully responsible for maintaining high levels of quality of service. Moreover, DSOs are increasingly empowered of forcing temporary modifications in DG injection profile (according to technical connection rules [37], [38]); on the other hand, no possibility is given to modify customers' power demand. In the existing literature, it is commonly assumed that the load absorptions depend on season and peak/low demand periods, but are constant during the one-hour time frame [39]-[41]. More realistically, we model the behavior of loads by different scenarios according to the needs of the customers, the zonal load time variation characteristics and the statistical variation of customers' power demands. These uncertainties affect the modeling and evaluation of the system capacity to accommodate DG units. Knowing these parameters helps in predicting the impact of DG on system planning, design and operation. In order to perform the probabilistic assessment of DG penetration, we embrace Monte Carlo simulation for its flexibility in representing the uncertainties connected to the multiple load conditions across time in power systems, and in propagating them to the estimation of the probability of technical constraint infringement. The paper is developed in the following way: after the Introduction, Section 2 describes the probabilistic 
load flow analysis, based on Monte Carlo simulations [42]-[47] for determining the electrical network capacity, in terms of the probability density function of the maximum DG power which is possible to install at each bus of a MV distribution networks without modifying the protection, control and automation systems. We carry out the DG penetration analysis by means of a power flow algorithm, customized for MV distribution networks through the simulation of On-Load Tap Changer (OLTC) transformer in the primary substation [48], which receives as input the hourly load values during one year (Section 3). We assess several loading scenarios that reflect the varying energy consumption at different times of the day and of the year (Section 4). The maximum DG power injection that the network can withstand in its present configuration is evaluated by connecting increasing amounts of DG to one bus at a time. The time variability of the maximum DG power is assessed: we compute the yearly probability of constraint violation, and the confidence intervals of the maximum DG power with respect to the operating limits. The methodology is applied to a MV radial distribution network in Section 5; finally, the results are compared with the DLF analysis.

\section{Methodology AND SIMULATION STEPS}

We embrace a probabilistic framework that accounts for the statistical variation of the loads throughout the year. To this aim, the input loads of the PLF reflect the average load profile of the two typical MVconnected loads: MV customers and MV/LV transformers supplying LV customers.

Fig. 1 visually exemplifies how uncertainties in the operating conditions impact electrical distribution networks by resorting to a simplified bi-dimensional view, where only two technical constraints are considered, i.e. line ampacity (thermal limits) and steady state voltage profile (supply voltage variation). If there is no variability in the customer's power demands, the DLF analysis exactly quantifies the system operating conditions (dot in Fig. 1). Conversely, when variability is considered, the electrical network may operate in multiple conditions (small solid line rectangle), some of which may exceed the operating limits (large dotted line rectangle): these particular operating conditions are represented by the shaded area. In this respect, the DLF analysis based, e.g., on average customers power demands (black dot), overlooks the possible constraint violations, whose probability is given by the probability of occurrence of operating conditions within the shaded area; this means that the idea of considering the worst case load and generation combination may be too pessimistic.

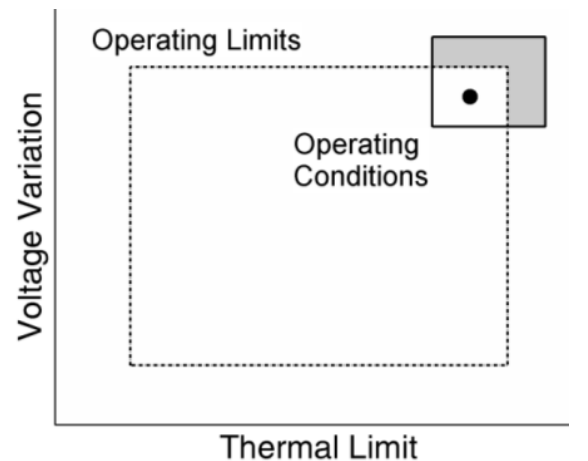

Fig. 1. Abstract representation of the $2 \mathrm{D}$ phase space of the electrical network. For clarity but with no loss of generality, only two dimensions are shown.

Moreover, DLF analysis with all customers' power requests being at their average, minimum and maximum values, not only implies unreasonable network conditions that never occur, but also introduces combinatorial problems and arbitrariness in assigning one of the three values to each customer's power request. The latter is especially true when the network size is large.

In this paper, we aim at overcoming the above limitations related to a deterministic load modeling. To this end, we embrace a probabilistic load modeling, and build probability distributions which describe the customers' power requests and fully exploit the hourly variability of field data. By sampling power values from probability distributions, we succeed in combining the possible power request values of all the 
costumers according to their occurrence probabilities. The Monte Carlo approach is used in order to estimate the probability density function of constraint violation associated to a specific DG unit taking into account the variability of the loads during each hour. In our analysis, for the sake of simplicity, the variability of DG is not considered. We assume that DG units run with full capacity according to the availability of primary source; the power injected into the grid is equal to rated power in order to maximize revenues.

The constraint violation probability due to $\mathrm{DG}$ is estimated by the evaluation of cumulative density functions (CDFs), eventually choosing the enforced limit on the basis of the accepted risk level. As a consequence, the final maximum DG power is selected on the basis of a given constraint violation probability. This parameter is defined by the DSO according to his willingness to accept a given risk level, which is managed by resorting to specific countermeasures, such as a temporary modulation/limitation of DG active power.

The network behavior is simulated via repeated load flow calculations. We investigate the maximum DG power that can be connected to each MV bus, $i$, within the range $[0,10] \mathrm{MW}$, the range for DG stated by [49], that considers as DG only the generation units lower than $10 \mathrm{MW}$.

According to the Nodal Hosting Capacity (NHC) approach, a new DG unit is simulated in a specific bus of a MV distribution grid: branch currents and bus voltages are computed by load flow calculations and compared with the operating limits. As long as no electric constraint is infringed, the bus $i$ is charged with increasing DG power in the above range (DG is assumed to have a power factor equal to 1). In order to calculate the NHC of the network, the procedure increases the DG power injected by $10 \mathrm{~kW}$ until one of the limits is violated. The maximum power injection which does not determine a violation quantifies the capability of the selected bus to accept DG power injections. Although the analysis is based on the presence of one generator at a time, the use of a very high ceiling for the "equivalent" generator, i.e. $10 \mathrm{MW}$, allows exploring the network limits when more realistic generators are connected.

The implementation of Monte Carlo simulations with repeated load flow calculations is detailed in Fig. 2. The LF calculations are embedded within a cycle that simulates many load scenarios for different realizations. This ensures that the maximum DG power is assessed spanning throughout the whole range of load variability; different load profiles are used for MV/LV substations and for direct MV customers, which have a narrow range of variation between peak and minimum demand (loads have a power factor equal to 0.9 ).

Three kinds of limits are taken into account by the procedure:

- line thermal limits; i.e., current limit of each branch;

- supply voltage variations for each bus;

- rapid voltage changes associated to sudden shift (e.g. trip) of DG power output.

\subsection{Line Thermal Limits}

The maximum DG power hosted at bus $i$ due to current constraints is limited by the approaching of critical thermal stresses in the network conductors. If the current exceeds the maximum capacity, there is premature wear and tear of the lines that affects the reliability of the conductors. In the present analysis, a 250 A limit is assumed for the ampacity of all branches taking into account common ratings of $\mathrm{MV}$ overhead lines and cables (e.g. a $185 \mathrm{~mm}^{2}$ aluminum) and the standard current settings for line relays in Italian MV grids. The maximum injection due to Line Thermal Limits (LTL) is usually dependent on the nominal voltage of the feeder and has a slight increase from top to bottom due to voltage increase, with local variations due to presence of loads. 


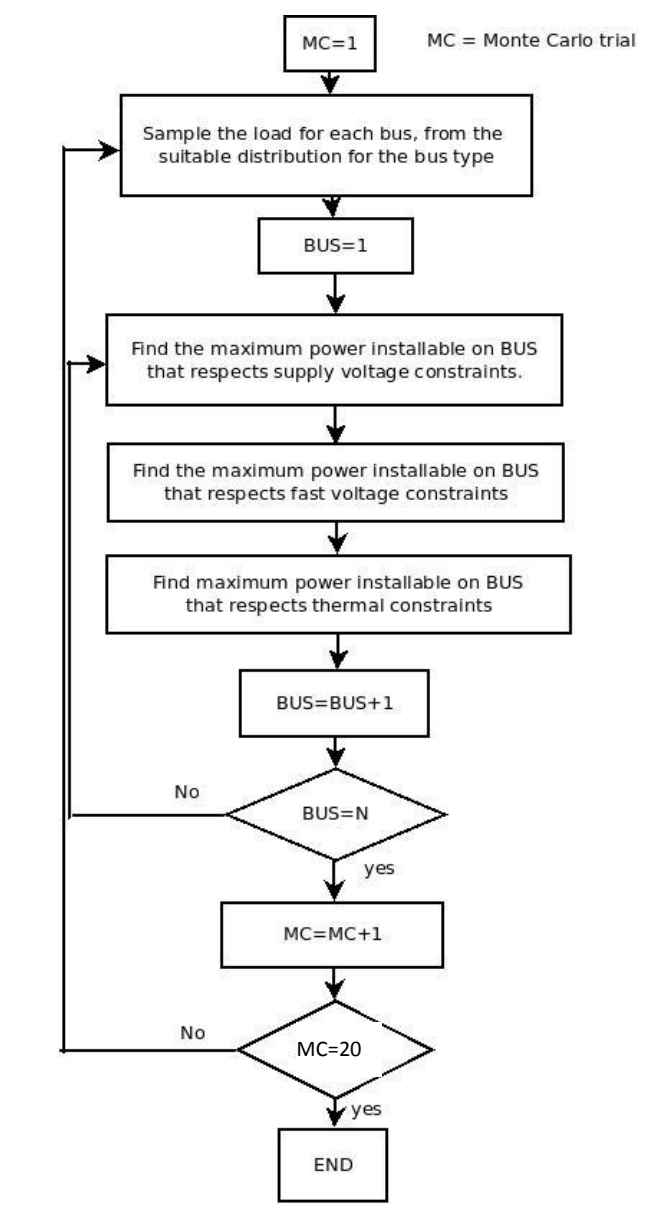

Fig. 2. Flow chart of the simulation steps for PLF.

\subsection{Supply Voltage Variations}

The installation of a generator causes the increase in steady state voltage value at the DG's PCC, and along the entire feeder. According to standard EN 50160 [51], the operating voltage of each bus must be between $90 \%$ and $110 \%$ of the nominal value. The upper limit could be violated (up to $115 \%$ ) for $1 \%$ of the time: this possibility is not exploited in the calculations, but could be exploited by the DSO as an additional margin for the cases in which a violation is accepted on a probabilistic basis. Voltage profile is controlled by means of an On OLTC transformer installed in the primary substation. The limit of installed DG power according to Supply Voltage Variation (SVV) is the value of the active power supplied by a generator that increases the voltage to the $110 \%$ of the nominal voltage.

\subsection{Rapid Voltage Changes}

A sudden generator (or load) disconnection causes a Rapid Voltage Change (RVC) within an electric network. According to the definition given in [50], RVCs are calculated by the comparison of two LF calculations, as differences between bus voltages obtained with and without the new DG unit in operation. A RVC value equal to $6 \%$ is accepted according to technical standard EN 50160 [51], although this limit cannot really be considered as a hard constraint. The maximum DG power installed in accordance with RVCs strongly depends on the short circuit power in the PCC, as discussed in [52].

\section{REgulated POWER FlOW}

The power flow equations model the electric network behavior, quantifying the power distribution along the network, the bus voltages, and the current flows in the branches, for a steady state network. The LF algorithm resorts to the Newton-Raphson method to calculate the electric variables [42]-[47], using Matlab tools [53]. 
Additionally, in order to simulate the actual voltage regulation by the OLTC transformer of primary substation, our customized LF [14], [48] requires $|V|$ of the MV busbar of the HV/MV transformer in primary substation to be equal to a fixed setpoint. This value, according to the practices adopted by DSOs, is determined in the condition of maximum load and no generation, in order to avoid possible infringements of the lower bound. As a consequence, it is calculated with a dedicated procedure developed in order to keep, for the maximum load, the operating voltage of each bus between $90 \%$ and $110 \%$ of the nominal value.

During operation, the transformation ratio $k$ of the HV/MV transformer will continuously adapt in order to maintain the desired voltage value $|V|$ at the MV primary busbars (PVQ bus). With this modification, $k$ becomes an additional variable of the regulated LF and is classified as the main unknown only on the bus corresponding to the MV bars. This bus type, which is usually a PQ-type bus, must maintain its $|\mathrm{V}|$ to a predetermined constant value; it therefore belongs to a particular category of buses, not classified in the classical load flow models, i.e. PVQ bus type.

In any of the MV distribution network there are only one balancing bus (HV bars), only one PVQ (MV bars) bus, and (n-2) PQ busses. In fact, at the busses belonging to different lines of the network, in addition to loads, there can be distributed generators, as they have been modeled for this work, introduce power $\mathrm{P}$ in the network with $\cos \varphi$ constant. The distribution networks buses are distinguished into the categories in Table 1, where $n$ indicates the total number of nodes of the generic distribution network.

TABLE 1

CATEGORIES OF BUSES IN A DISTRIBUTION NETWORK, DEPENDING ON THE TYPE OF UNKNOWNS CONSIDERED IN THE "AD HOC" LOAD FLOW

\begin{tabular}{clcc}
\hline \hline Bus Type & Number of buses & Known variables & Unknown variables \\
PQ & $n-2$ & $(P, Q)$ & $(V, \delta)$ \\
PVQ & 1 & $(P, Q, V)$ & $(\delta, k)$ \\
Balance Bus & 1 & $(V, \delta)$ & $(P, Q)$ \\
\hline
\end{tabular}

Considering the transformation ratio $k$, the active and reactive equations of our LF take the following general form (where with $n$ indicates the total number of nodes of the generic distribution network):

$$
\left\{\begin{array}{l}
P_{p}\left(V_{q}, \delta_{q}, k_{q}\right)=k_{p} V_{p} \cdot \sum_{q=1}^{n}\left[Y_{p q} \cdot k_{q} V_{q} \cdot \cos \left(\delta_{p}-\delta_{q}-\theta_{p q}\right)\right] \\
Q_{p}\left(V_{q}, \delta_{q}, k_{q}\right)=k_{p} V_{p} \cdot \sum_{q=1}^{n}\left[Y_{p q} \cdot k_{q} V_{q} \cdot \sin \left(\delta_{p}-\delta_{q}-\theta_{p q}\right)\right]
\end{array}\right.
$$

In these equations, $k$ is constant and equal to 1 for all buses except for the PVQ bus. Therefore, its value is irrelevant for all $(n-1)$ network buses other than the PVQ. $k$ varies by steps of 0.01 in order to keep the desired $|V|$ at the MV busbar of primary substation (the PVQ bus).

Given a network with $n$ buses, we solve iteratively the system of equations by converging, with an "ad hoc" procedure which is tailored to the MV distribution networks [14], [48]. The application of the load flow model set of a distribution network consisting of $n$ buses is thus to solve a problem consisting of $(2 n-2)$ equations in $(2 n-2)$ unknowns.

\section{INPUT DATA}

To simulate the network configuration during normal operation, we resort to the empirical power curves representing the time profile (during one year) of the power withdrawn by MV direct customers (Fig. 3) and by MV/LV transformers (Fig. 4). 


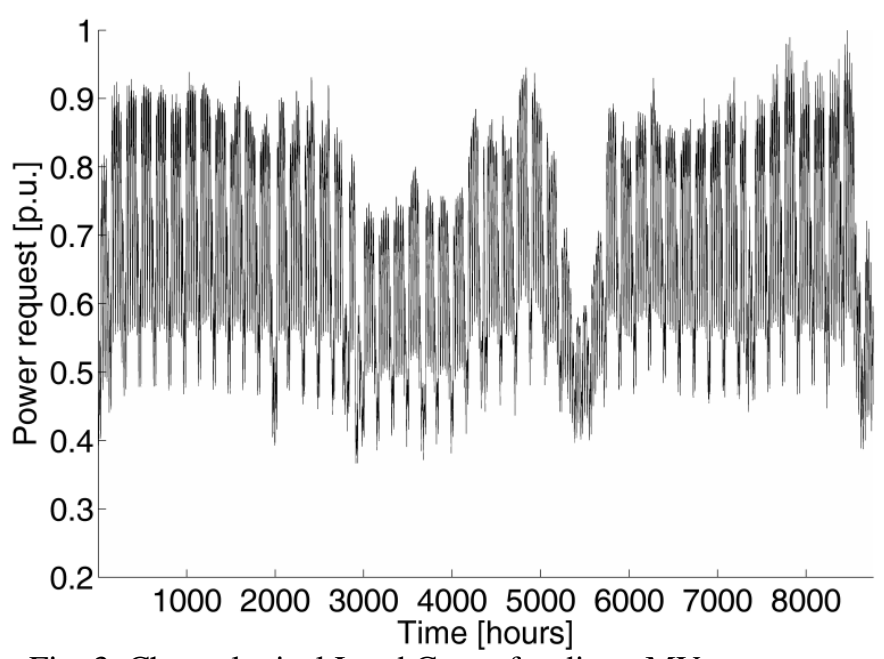

Fig. 3. Chronological Load Curve for direct MV customers.

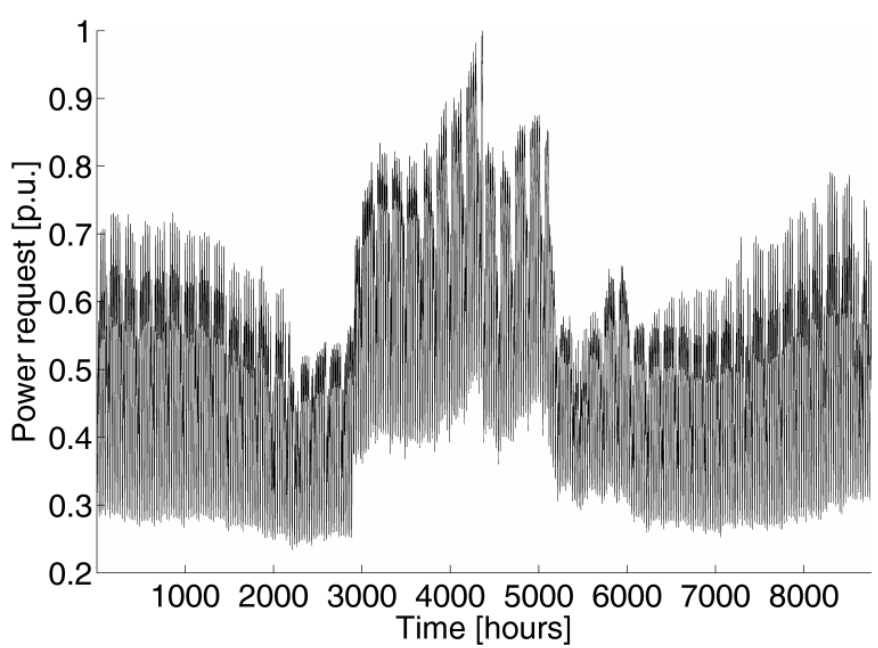

Fig. 4. Chronological Load Curve for MV/LV transformers.

These curves have been derived from the data made available by the Italian Transmission System Operator (TSO), Terna, which represent the demand profiles of the two different MV load classes on a national basis. Given that the two loads' classes use energy at different rates during time, it is necessary to model the relevant power absorption by two different load curves.

The power demand varies periodically both within the day and within the year. Therefore, time bands with homogeneous electric power demands can be identified. The load values are arranged into subsets with nearly homogeneous power consumption, and the optimum number and size of these subsets were determined by a cluster analysis [14] based on the specifications given by the Italian Energy Regulator [54]. Table 2 reports the definition of the time bands and the associated time frames. The hourly values of power requests are grouped into six homogeneous hourly bands for each month amounting to 72 yearly time bands. In standard DLF, the clustering procedure aims at reducing the number of simulations and the computational time, because the average load of the time band is assumed to describe the loading scenario within the time band.

TABLE 2

TIME BANDS AS DEFINED BY REGULATORY ORDER 181/06 [54]

\begin{tabular}{cccc}
\hline \hline Time band & Acronym & Description & Time frame \\
\hline Workdays & $\mathrm{f} 1$ & Peak hours & workdays: 8 AM - 7 PM \\
Workdays & $\mathrm{f} 2$ & Intermediate demand & workdays: 7 - 8 AM \& 7-11 PM \\
Workdays & $\mathrm{f} 3$ & Out-of-peak hours & workdays: 11 PM - 7 AM \\
Saturdays & $\mathrm{f} 4$ & Intermediate demand & Saturday: 7 AM - 11 PM \\
Saturdays & $\mathrm{f5}$ & Out-of-peak hours & Saturday: 11 PM - 7 AM
\end{tabular}


In our study, we use a probabilistic approach to capture the variability of each load of the distribution network, based on the data available at a national level. Starting from the two chronological load curves (Fig. 3 and 4), we group the 8760 hourly load values into 72 time bands, six homogeneous hourly bands for each month (Table 2). The time bands for each month contain three types of "equivalent days", i.e. workdays, Saturdays and holidays (including Sundays). Each type of "equivalent day" is further defined by different numbers of points: weekdays by three points (bands f1, f2 and f3, Table 2), Saturdays by two points (bands f4 and f5, Table 2), holidays and Sundays by one point (band f6, Table 2).

The 72 clusters of hourly load values identify probability distributions for each time band and for each of the two load classes, i.e. MV direct customers and by MV/LV transformers. We build 144 distributions that describe customers' power requests throughout the year. These distributions are used for sampling the power request values during the appropriate time band.

Fig. 5 shows the six cumulative probability distributions that describe the load requests during the six time bands of January for direct MV customers. We notice that these load distributions which are built from time bands of nearly homogeneous power consumption show some variability.

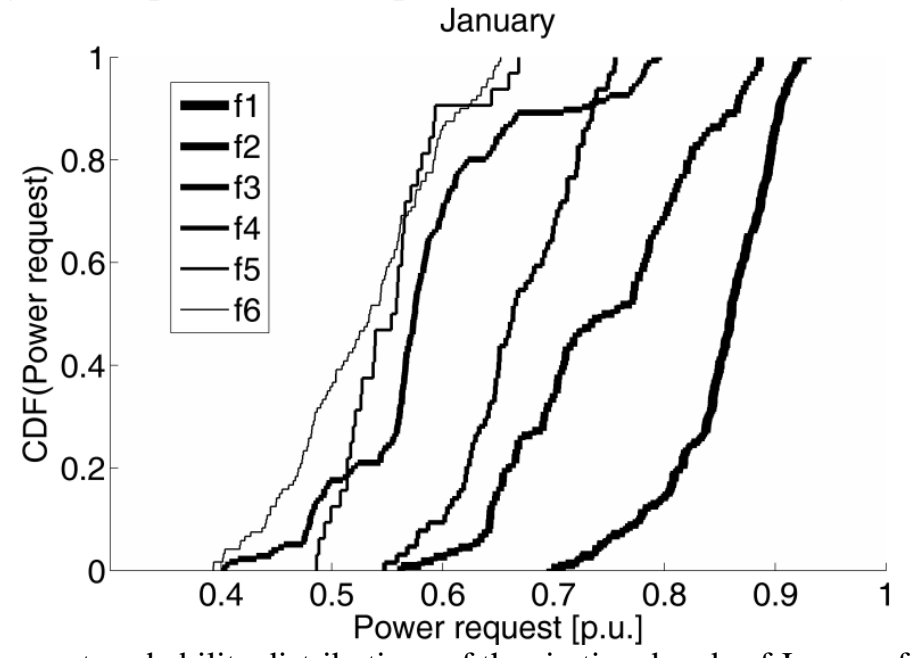

Fig. 5. The cumulative power request probability distributions of the six time bands of January for direct MV customers. These distributions are built from the chronological load curves of January (Fig. 3).

The PLF approach is capable of capturing the full variability of power requests within the time bands. On the contrary, the DLF approach based on average power consumption assumes that the power request values in the time bands can be approximated by their average values.

Probability distributions similar to those in Fig. 5 are built for every month and for the two load classes. Fig. 6 plots the mean values of the 144 probability distribution of the power requests for direct MV customers and MV/LV substations. These values identify the two Time Band Curves which are employed in the DLF analysis based on average power consumption. 


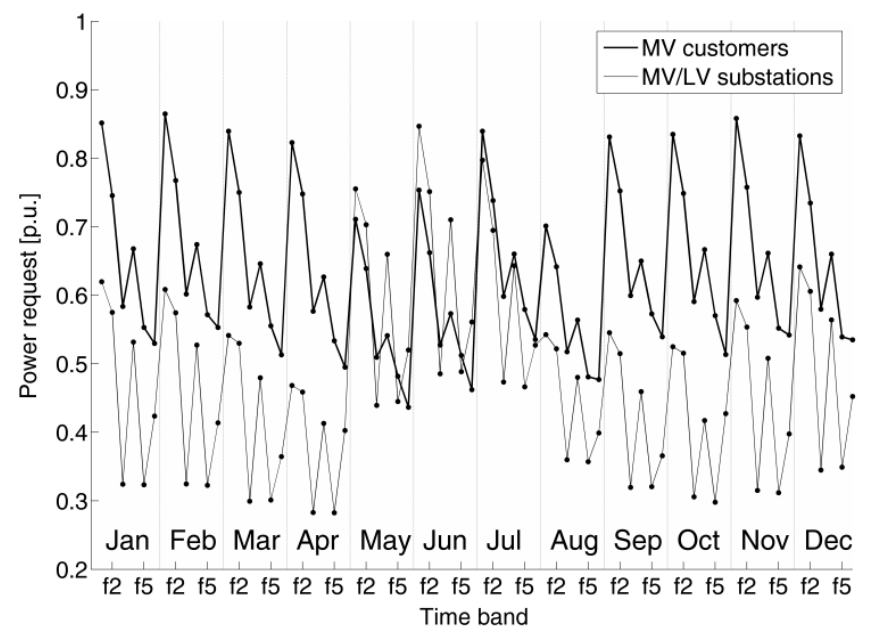

Fig. 6. Time Band Curves of the power requests for direct MV customers and MV/LV substations. Each point identifies the mean value of the power requests distributions for each time band and month.

Within our PLF approach, the load configurations of the direct MV customers and of MV/LV substations are sampled from the power request probability distributions of the appropriate time band. The sequential Monte Carlo (MC) trials differ from one another because different load scenarios are realized. For each MC trial, we identify the maximum DG power that can be connected to every bus with respect to the different limits, and quantify the maximum DG power within the time bands.

\section{RESULTS}

The methodology for maximum DG power allocation on a single bus has been applied to a real Italian MV network with 274 buses distributed in 4 feeders; this sample network is derived from a huge data sample of the MV Italian system.

We run $20 \mathrm{MC}$ trials for each time band (a total of $1440 \mathrm{MC}$ trials) to obtain a full representation of the customers power demands. Twenty MC trials for each time band yield 60 maximum DG power values, 20 determined by Line Thermal Limits, 20 by Supply Voltage Variations, and 20 by Rapid Voltage Changes, for each time band. We take the average value of the 3 groups of 20 DG power values, and obtain 3 values; the smallest of the three represents the more stringent constraint. Repeating this procedure for 72 time bands, we get 72 DG power values, the minimum of which identifies the most limiting time band for a bus. Likewise, we identified the most limiting time band for each bus, and the bus with the smallest installable DG power given by the most limiting constraint.

Looking in more details at the constraint violation, we found that Rapid Voltage Changes are the most limiting constraints, and indeed most of the 274 buses reach their maximum allowed DG due to this limit. Rapid Voltage Changes are little sensitive to load variations. This appears especially true when we account for variations within time bands of homogeneous customers' power demands. For Rapid Voltage Change constraints, DLF analysis based on average demands within the time bands yields the same results of the PLF analysis.

Based on the fact that Rapid Voltage Change constraints are not considered by the quality of service European standards as a hard constraint [51], we explore further the NHC of each bus focusing on the other two technical constraints: Line Thermal Limit and Supply Voltage Variations.

They display larger variability with respect to customers' power demands in the time bands. We found that the maximum installable DG power has large variability within the network, and all the buses show at least one constraint violation in the test range $[0,10] \mathrm{MW}$ of DG power.

The analysis is carried out for all the four feeders of the network, finding the most limiting bus and constraint within the feeders. Table 3 summarizes the maximum installable DG power distribution for the most limiting buses of each feeder and the most limiting time band. 
TABLE 3.

MOST LIMITING BUSES

\begin{tabular}{ccccc}
\hline \hline & Feeder & Feeder & Feeder & Feeder \\
& 1 & 2 & 3 & 4 \\
\hline Bus & 3 & 48 & 162 & 214 \\
Month & 4 & 4 & 4 & 4 \\
Time band & f5 & f5 & f5 & f5 \\
Constraint & LTL & SVV & SVV & SVV \\
DLF [MW] & 6.77 & 1.41 & 3.79 & 3.42 \\
25 ${ }^{\text {th }}$ perc. & 6.76 & 1.41 & 3.76 & 3.41 \\
75 $^{\text {th }}$ perc. & 6.79 & 1.44 & 3.82 & 3.44 \\
PLF Std dev. & 0.01 & 0.02 & 0.03 & 0.02 \\
PLF Mean & 6.77 & 1.42 & 3.79 & 3.42 \\
\hline
\end{tabular}

From Table 3, we notice that bus 48 in the second feeder shows the smallest average maximum installable DG power. This limiting value corresponds to the violation of the Supply Voltage Variation constraint during the f5 time band of April. We notice that the use of $20 \mathrm{MC}$ trials is enough to obtain a relative standard error of $1.6 \%$ at worst (Table 3, bus 48). Moreover, the estimates are reliable up to the tenth of $\mathrm{kW}$ in the worst case (Table 3, bus 162) according to the Leading-Digit Rule omitting digits which are 0.117meaningless [55]. This behavior is explained by the fact that the distributions of loads built from time bands of homogeneous customers power demands show moderate variability (Fig. 5). In some cases, the output of PLF analysis within the single time band coincides with the DLF analysis.

\subsection{Distributions of the maximum DG power for bus 48}

To achieve a secure DG penetration, we must take into account the yearly variability of the customers power demands that impacts the constraint violation risk throughout the year. To this aim, Fig. 7 quantifies the yearly variability of the maximum DG power within the time bands for the most limiting bus, i.e., bus 48. The all-year, time band analysis is summarized by this empirical CDF that describes the yearly probability of a constraint violation if a certain amount of power is generated at bus 48 . We build Fig. 7 as follows. For bus 48, we scan from the bottom to the top the 1440 maximum DG power values, and count the number of constraint violations, $N V_{i}$, for the $i$-th power step, $D G_{i} \in[0,10] \mathrm{MW} . N V_{i}$ is the number of maximum DG power values below the $i$-th power step, $D G_{i}$. We weigh each violation according to the time width of the time band in which it occurs. The yearly probability of a constraint violation for $D G_{i}$ is the sum of the $N V_{i}$ weights. Fig. 7 displays the yearly violation probability of the three constraints with respect to increasing values of DG power of bus 48 .

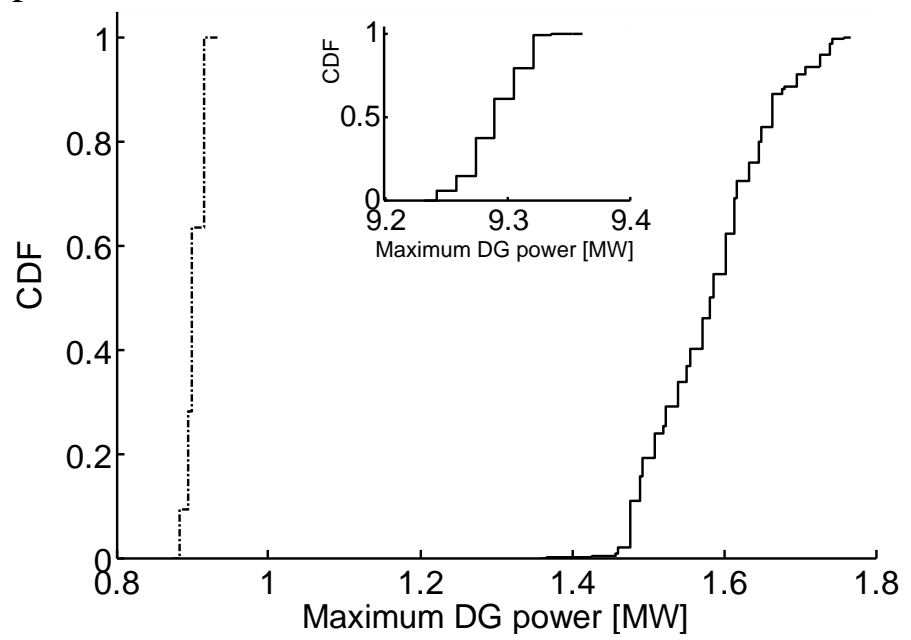

Fig. 7. The yearly probability of SVV (solid line), Rapid Voltage Changes (dashed line), and LTL (inset) constraint violation as a function of the maximum DG connected to bus 48 . The CDFs quantify the yearly probability to violate the constraints if a certain amount of power is generated at bus 48 . 
Fig. 7 quantifies the probability of constraint violation as a function of the installed DG power, but loses the information on the variability of the maximum DG power within the time bands. To this aim, Fig. 8 shows the empirical CDFs of the maximum DG power connected to bus 48 with respect to SVV, LTL and Rapid Voltage Changes, during the most limiting time band for bus 48, i.e., April - f5.

The CDFs in Fig. 7 and 8 allow identifying probabilistic safety margins for DG power allocation with respect to the three constraints. For comparison, Fig. 8 displays the CDFs of the maximum DG power distribution for bus 48 in the most limiting time band, i.e. April-f5. The CDFs in Figure 8 display small variability because load values in April-f5 time band are rather homogeneous.

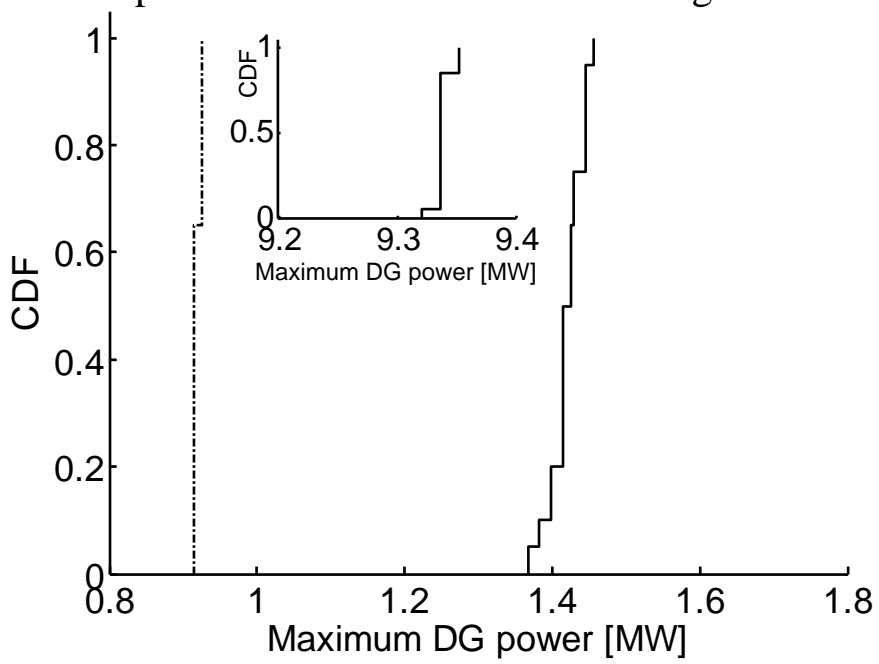

Fig. 8. The probability of SVV (solid line), Rapid Voltage Changes (dashed line), and LTL (inset) constraint violation as a function of the maximum DG connected to bus 48 in the most limiting time band, i.e., April - f5. The CDFs quantify the probability to violate the constraints during April - f5 if a given amount of power is generated at bus 48 .

An evaluation based solely on the worst time band analysis (Fig. 8) is a conservative one, because it fails to account for the whole variability information that characterizes the entire year (Fig. 7). For example, if we want to limit the yearly probability of SVV violations to $5 \%$ in bus 48 , we must limit the DG connected to bus 48 to $1.48 \mathrm{MW}$ (Fig. 7). On the contrary, if we want to limit the probability of SVV violations to $5 \%$ in bus 48 based solely on the worst time band analysis (Fig. 8), we must limit the DG connected to bus 48 to 1.37 MW. The DLF analysis yields the value 1.41 MW of the maximum DG power (Table 3). For bus 48, the all-year probabilistic approach allows the additional generation of $8 \%$ DG compared to the worst-timeband approach, and of $4.5 \%$ DG power compared to the deterministic approach. Obviously, larger improvements are obtained if the limit on the violation probability is relaxed.

To gain the overall perspective of the maximum output DG power for the entire network, we computed the CDFs of the maximum DG power connected to every bus with respect to the Supply Voltage Variation, Rapid Voltage Change and Line Thermal Limit, during their most limiting time band. For comparison, the CDFs can be summarized by the 0.05 and 0.95 percentiles, which identify confidence intervals for the maximum DG power. Fig. 9 displays a two dimensional plot of the entire network with respect to SVV and LTL. The rectangles identify 2-dimensional probabilistic margins for LTL and SVV constraint violations for each bus. The left and the lower sides of the rectangles identify the $95 \%$ margins (or 5\% probability limits) with respect to Supply Voltage Variations and Line Thermal Limits, respectively. The area of the rectangles provides an indication of the impact that uncertainty in customers power demands has on the maximum DG power that can be connected. 


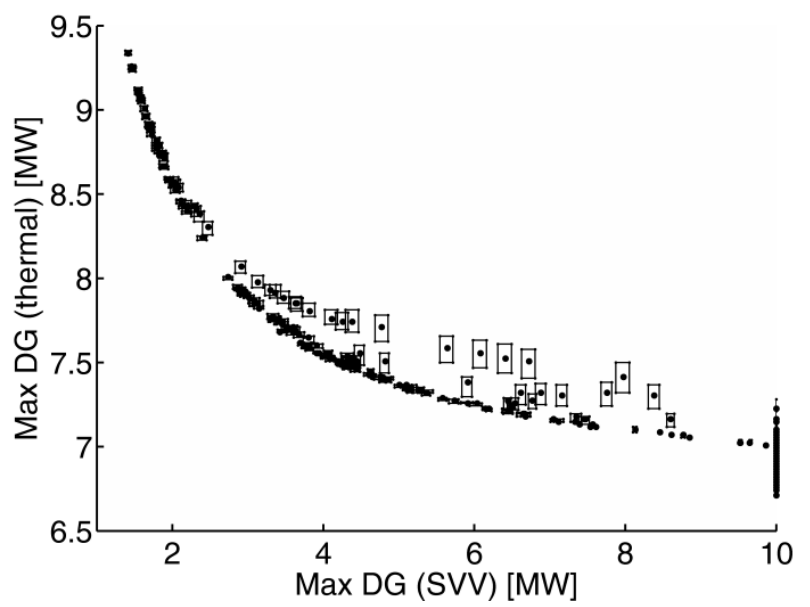

Fig. 9. Rectangles of the 0.05 and 0.95 percentiles of the maximum DG power that can be connected to all the buses in their most limiting time band, with respect to Supply Voltage Variations and thermal limits. Each rectangle corresponds to a bus. Dots mark the DLF results.

All buses encounter at least thermal limits before $10 \mathrm{MW}$, but all of them can accommodate at least $1 \mathrm{MW}$. LTL constraints do not limit the maximum DG power beyond roughly 6.5 MW; conversely, Supply Voltage Variation limits are bottlenecks for many buses. From Fig. 9, we notice that several buses show a large variability in the maximum DG power. In these cases, the DLF DG penetration analysis does not provide exhaustive indications: installing a DG as prescribed by DLF (dots in Fig. 9) may lead to constraints infringements in realistic customers power demand configurations (rectangles in Fig. 9).

The results show that Line Thermal Limits affect mainly the buses close to the primary substation, whereas Supply Voltage Variations (and Rapid Voltage Changes) limits are more significant for buses far from primary substation. Apart from Rapid Voltage Changes (that according to EN 50160 cannot be considered as a hard constraint), Supply Voltage Variation limits can be overcome by a proper voltage regulation strategy, involving an active participation of DG units (in the perspective of smart grids); on the contrary, Line Thermal Limits constraints really represent the network structural characteristics and are related to the cross sections of MV overhead lines and cables. Such structural limits cannot be overcome unless significant investments are put in place (traditional network expansion).

\section{DISCUSSION}

NHC analyses are a conventional approach to evaluate the capability of a network of accepting one equivalent generator in one bus at a time. Similar to the optimization methods and the deterministic NHC methods, the combination of NHC and MC simulation allows the identification of the most critical constraints for each bus and points towards possible strategies to overcome them, e.g. by voltage regulation. On the other hand, the flexibility of the hybrid NHC and MC method enables the identification of probabilistic safety margins with respect to the installed size of DG in terms of the likelihood of constraint violation. Furthermore, this method allows the quantification of the additional DG power that can be connected to one bus if a larger value of the constraint violation probability can be tolerated. This is equivalent to relaxing one constraint in the optimization method. Although the allocation of multiple DG units can also be accomplished by the hybrid NHC and MC method, the maximum DG power is conditioned on the current output of other DG and a consistent procedure for quantifying the hosting capacity should be devised in this situation. In this respect, the optimization method can adapt to multiple DG units allocation in a straightforward way. On the contrary, power demands at each load point can be modeled as independent during each time step in the hybrid NHC and MC method, whereas they are modulated by the same load factor in other approaches. Moreover, due to the inherent combinatorial nature of DG siting and sizing, the optimization methods cannot account for multiple load conditions across time for network planning. 
It has to be highlighted that the limits found by the NHC approach are not aimed at the actual connection of a specific DG unit: this task has to be faced by the DSO during the connection procedure, taking into account also the possible presence of other units already connected.

Moving from the nodal approach to the system approach, problems related to interface protection relays and reverse power-flow time (percentage of time during which power flows from medium to high voltage due to a surplus in power production, i.e. a larger amount of power is injected in the distribution network by DG, compared to the local demand), were detected, that would prevent the full exploitation of the distribution network, up to the system hosting capacity (SHC). For each primary substation, SHC is defined as the maximum DG capacity that it is possible to connect with no traditional network expansion, while still respecting the thermal limits of the network.

The only way to meet these challenges is the adoption of innovative solutions: these will conduct to a more efficient planning and operation of the grid, by means of improved automation and control of network components and end-users' participation (smart grids) [56].

\section{CONCLUSIONS}

The power demand from the loads of MV distribution networks is affected by variability, which reflects on the power flows and voltage profile throughout the feeders. Within a DG penetration assessment, we presented a probabilistic load flow analysis using Monte Carlo simulations to account for load variability. We quantified the maximum DG power that can be connected to each bus of the distribution network without modifying protection, control and automation systems. We simulated the variability of the customers power demands, and determined the maximum DG power for each bus of the network that does not violate the operating limits. This indication is useful to DSOs in drawing up an optimal expansion plan for the MV distribution network, in order to cope with the increasing connection requests, while ensuring a proper and efficient functioning of the network. The problem involves several factors, not just technical (rated power, characteristics of the electric network, technological innovations, etc.) but also managerial (economic impact of new infrastructures, impacts due to regulation, impacts due to authorization procedures, cancellations and failure to carry out installations, etc.). The adoption of PLF calculations can provide the DSOs with a technical solution based on a possible range of risk that takes into account the variability of loads. The DSO could decide to connect a quantity of DG greater than the DLF Nodal Hosting Capacity (accepting a higher risk) for a few hours of year; during this period the DSO could rely on temporary constraint relaxations (as the ones given for overvoltage values up to $115 \%$ in EN 50160). In the future, DSOs will be able to implement innovative solutions such as limitation/modulation of active power injected by DG (or eventually storage systems) in order to allow the secure operation of the system. We claim that the PLF approach can be more appropriate than the DLF one, due to the realistic representation of the variability of the customers' loads, and the capability of providing probabilistic margins with respect to constraint violation. In more detail, the results show the buses of the network where the load variability can significantly affect the amount of DG.

Finally, as a possible research perspective, the exploitation of probabilistic margins could be a valid solution towards a better assessment (by the use of probabilistic analyses) of service reliability, availability and quality of MV networks with high penetration of DG.

\section{REFERENCES}

[1] "EU Directive 2009/28/CE", European Parliament and Council in December 2008 agreed the 20-20-20 package and became law in June 2009.

[2] M. Delfanti, M. Merlo, G. Monfredini, V. Olivieri, M. Pozzi, A. Silvestri, "Hosting Distributed Generation on Italian MV networks: towards smart grids", 2010 14th International Conference on Harmonics and Quality of Power (ICHQP), 26-29 Sept. 2010, pp.1-6.

[3] G. Pepermans, J. Driesen, D. Haeseldonckx, R. Belmans, W. D'haeseleer, Distributed generation: Definition, benefits and issues, Energy Policy Volume 33, Issue 6, April 2005, Pages 787-798.

[4] P. Chiradeja, R. Ramakumar, An approach to quantify the technical benefits of distributed generation, IEEE Transactions on Energy Conversion, Volume 19, Issue 4, December 2004, Pages 764-773. 
[5] M. Thomson, D.G. Infield, Impact of widespread photovoltaics generation on distribution systems, IET Renewable Power Generation, Volume 1, Issue 1, 2007, Pages 33-40.

[6] R.A. Shayani, M.A.G. de Oliveira, Photovoltaic Generation Penetration Limits in Radial Distribution Systems, IEEE Transactions on Power Systems, vol. 26, no. 3, Aug. 2011.

[7] J.A.P. Lopes, N. Hatziargyriou, J. Mutale, P. Djapic, N. Jenkins. Integrating distributed generation into electric power systems: A review of drivers, challenges and opportunities, Electric Power Systems Research, Volume 77, Issue 9, July 2007, Pages 1189-1203.

[8] P.P. Barker, R.W. De Mello, Determining the impact of distributed generation on power systems: Part 1 - Radial distribution systems, Proceedings of the IEEE Power Engineering Society Transmission and Distribution Conference Volume 3, 2000, Pages 1645-1656.

[9] M. Thomson, D.G. Infield, Network Power-Flow Analysis for a High Penetration of Distributed Generation ; IEEE Transactions on Power Systems, Aug. 2007, Volume 22, Issue 3.

[10] J. Deuse, S. Grenard, M.H.J. Bollen, EU-DEEP integrated project - Technical implications of the "hosting-capacity" of the system for DER, Int Journal of Distributed Energy Resources, Vol.4, No.1 (2008), pp.17-34.

[11] N. Etherden, M.H.J. Bollen; "Increasing the hosting capacity of distribution networks by curtailment of renewable energy resources", 2011 IEEE Trondheim PowerTech, 19-23 June 2011, pp.1-7.

[12] C.J. Dent, L.F. Ochoa, G.P. Harrison; "Network distributed generation capacity analysis using OPF with voltage step constraints", IEEE Transactions on Power Systems, vol.25, no.1, pp.296-304, Feb. 2010.

[13] Ma Xiufan, Li Haimei, "Distribution network planning based on yearly load duration curve", 20112 nd International Conference on Artificial Intelligence, Management Science and Electronic Commerce (AIMSEC) 8-10 Aug. 2011, pp.39263930.

[14] M. Delfanti, M.S. Pasquadibisceglie, M. Pozzi, M. Gallanti, R. Vailati "Limits to Distributed generation on Italian MV networks" Electricity Distribution - Part 1, 2009. CIRED 2009. 20th International Conference and Exhibition on , vol., no., pp.1-4, 8-11 June 2009.

[15] Kumar A, Gao W. Optimal distributed generation location using mixed integer non-linear programming in hybrid electricity markets. IET Gener Transm Distrib 2010;4(2):281-98.

[16] S. McCusker, B. Hobbs, and Y. Ji, "Distributed utility planning using probabilistic production costing and generalized benders decomposition,” IEEE Trans. Power Syst., vol. 17, no. 2, pp. 497-505, May 2002.

[17] W. El-Khattam, Y. Hegazy, and M. Salama, "An integrated distributed generation optimization model for distribution systemplanning,” IEEE Trans. Power Syst., vol. 20, no. 2, pp. 1158-1165, May 2005.

[18] Khyati DM, Roy R. Enhancement of loading capacity of distribution system through distributed generator placement considering techno-economic benefits with load growth. Int J Electr Power Energy Syst 2014;54:505-15.

[19] Moradi MH, Eskandari M. A hybrid method for simultaneous optimization of DG capacity and operational strategy in microgrids considering uncertainty in electricity price forecasting. Renewable Energy 2014;68:697-714.

[20] Borges Carmen LT, Falcão Djalma M. Optimal distributed generation allocation for reliability, losses and voltage improvement. Int J Electr Power Energy Syst 2006;28(6):413-20.

[21] Singh RK, Goswami SK. Optimum allocation of distributed generations based on nodal pricing for profit, loss reduction, and voltage improvement including voltage rise issue. Int J Electr Power Energy Syst 2010;32(6):637-44.

[22] Falaghi H, Singh C, haghifam M-R, Ramezani M. DG integrated multistage distribution system expansion planning. Elect Power Energy Syst 2011;33:1489-97.

[23] Foster, J. D., Berry, A. M., Boland, N., Waterer, H. Comparison of Mixed-Integer Programming and Genetic Algorithm Methods for Distributed Generation Planning. IEEE Trans. Power Syst 2014;29(2):833-43.

[24] M.H.J. Bollen and F. Hassan Integration of Distributed Generation in the Power System - IEEE Press Series on Power Engineering, New York; Wiley-Blackwell, 2011.

[25] Abdi Sh, Afshar K. Application of IPSO-Monte Carlo for optimal distributed generation allocation and sizing. Elect Power Energy Syst 2013;44: 786-97.

[26] Mendez VH, Rivier J, de la Fuente JI, Gomez T, Arceluz J, Marin J, Madurga A. Impact of distributed generation on distribution investment deferral. Elect Power Energy Syst 2006;28: 244-52.

[27] Vallée F, Klonari V, Lisiecki T, Durieux O, Moiny F, Lobry J. Development of a probabilistic tool using Monte Carlo simulation and smart meters measurements for the long term analysis of low voltage distribution grids with photovoltaic generation. Elect Power Energy Syst 2013;53: 468-77.

[28] S. Conti, S. Raiti, Probabilistic load flow using Monte Carlo techniques for distribution networks with photovoltaic generator, Solar Energy, Volume 81, Issue 12, December 2007.

[29] M.R. Haghifam, A. Hadian "Distribution system adequacy assessment with consideration of stochastic distributed generation," Probabilistic Methods Applied to Power Systems (PMAPS), 2010 IEEE 11th International Conference on , vol., no., pp.553-557, 14-17 June 2010

[30] C.S. Indulkar "Monte Carlo Planning Technique for Renewable Energy Sources," Power System Technology and IEEE Power India Conference, 2008. POWERCON 2008. Joint International Conference on , vol., no., pp.1-7, 12-15 Oct. 2008

[31] Y. G. Hegazy, M.M.A. Salama, A.Y. Chikhani, Adequacy assessment of distributed generation systems using Monte Carlo Simulation, IEEE Transactions on Power Systems, Feb 2003, Volume 18, Issue 1 
[32] Banerjee B, Islam SM. Reliability based optimum location of distributed generation. Elect Power Energy Syst 2011;33:1470-8.

[33] Mokryani G, Siano P. Evaluating the integration of wind power into distribution networks by using Monte Carlo simulation. Elect Power Energy Syst 2013;53:244-55.

[34] G. Celli, E. Ghiani, S. Mocci et al. "A multiobjective evolutionary algorithm for the sizing and siting of distributed generation", IEEE Trans. Power Syst. , 2005, pp. 47-55

[35] V.H.M. Quezada, J.R. Abbad, T.G.S. Roman "Assessment of energy distribution losses for increasing penetration of distributed generation," Power Systems, IEEE Transactions on , vol.21, no.2, pp. 533- 540, May 2006

[36] M. Delfanti, D. Falabretti, M. Merlo "Losses assessment on distribution networks in presence of dispersed generation", CIRED Workshop 2012, pp.1-4, 29-30 May 2012.

[37] Italian Electrotechnical Committee (CEI), 2012, Technical standard CEI 0-21 "Reference technical rules for the connection of active and passive users to the LV electrical Utilities", Second Edition, in Italian, CEI, Milan (in Italian).

[38] VDEW 2004 Private electricity generation systems at the low voltage grid - Guideline for connecting and operating of distributed generation systems on the low voltage grid, 4 August 2001.

[39] Falaghi H, Singh C, Haghifam M-R, Ramezani M. DG integrated multistage distribution system expansion planning. Elect Power Energy Syst 2011;33:1489-97.

[40] Mistry KD, Roy R. Enhancement of loading capacity of distribution system through distributed generator placement considering techno-economic benefits with load growth. Elect Power Energy Syst 2014;54:505-15.

[41] Barin A, Pozzatti LF, Canha LN, Machado RQ, Abaide AR, Arend G. Multiobjective analysis of impacts of distributed generation placement on the operational characteristics of networks for distribution system planning. Electr Power Energy Syst 2010;32(10):1157-64.

[42] M.V.F. Pereira, M.E.P. Maceira, G.C. Oliveira, L.M.V.G. Pinto, Combining analytical models and Monte Carlo techniques in probabilistic power system analysis, IEEE Transactions on Power Systems, Volume 7, Issue 1, February 1992, Pages 265272 .

[43] Stefopoulos GK, Meliopoulos AP, Cokkinides GJ. Probabilistic power flow with non-conforming electric loads. Elect Power Energy Syst 2005;27:627-34.

[44] A.P. Meliopoulos, Sakis, Cokkinides, J. George, Chao, Xing Yong, New probabilistic power flow analysis method IEEE Transactions on Power Systems, Volume 5, Issue 1, February 1990, Pages 182-190.

[45] Papaefthymiou G, Schavemaker PH, van der Sluis L, Kling WL, Kurowicka D, Cooke RM. Integration of stochastic generation in power systems. Elect Power Energy Syst 2006;28:655-67.

[46] Gallego LA, Padilha-Feltrin A. Power flow for primary distribution networks considering uncertainty in demand and user connection. Elect Power Energy Syst 2012;43:1171-8.

[47] A. Sankarakrishnan, R. Billinton, Sequential Monte Carlo simulation for composite power system reliability analysis with time varying loads, IEEE Transactions on Power Systems, Volume 10, Issue 3, August 1995, Pages 1540-1545.

[48] Delfanti, M., Merlo, M., Pozzi, M., Olivieri, V., Gallanti, M. "Power flows in the Italian distribution electric system with dispersed generation” 2009 IET Conference Publications, (550 CP).

[49] DIRECTIVE 2003/54/EC OF THE EUROPEAN PARLIAMENT AND OF THE COUNCIL of 26 June 2003 concerning common rules for the internal market in electricity and repealing Directive 96/92/EC.

[50] International Electrotechnical Commission (IEC), 2008, Technical Standard IEC 61000 "Electromagnetic compatibility (EMC) - Part 4-30: Testing and measurement techniques - Power quality measurement methods", Edition 2.0, IEC, Geneva, pp. 1-130.

[51] CENELEC EN 50160, Voltage Characteristics of Electricity Supplied by Public Distribution Systems, 2010.

[52] V. Allegranza, A. Ardito, E. De Berardinis, M. Delfanti; L. Lo Schiavo: Assessment of short-circuit power level in HV and MV networks with respect to Power Quality, Electricity Distribution - Part 1, 2007. CIRED 2007. 19th International Conference and Exhibition on , vol., no., pp.1-5, 21-24 May 2007.

[53] R.D. Zimmerman, C.E. Murillo-Sánchez, R.J. Thomas, MATPOWER: Steady-State Operations, Planning, and Analysis Tools for Power Systems Research and Education, Power Systems, IEEE Transactions, on February 2011 , pages 12 - 19.

[54] Regulatory Order 181/06 of Italian Energy Regulator. "Update of time bands as of January 1 $1^{\text {st }}$, 2007” (in Italian), January 2007.

[55] W. T. Song and B. W. Schmeiser, “Omitting Meaningless Digits in Point Estimates: The Probability Guarantee of LeadingDigit Rules," Oper. Res., vol. 57, no. 1, pp. 109-111, 2009.

[56] L. Lo Schiavo, M. Delfanti, E. Fumagalli, V. Olivieri "Changing the regulation for regulating the change: Innovation-driven regulatory developments for smart grids, smart metering and e-mobility in Italy”, Energy Policy, ISSN 0301-4215, 10.1016/j.enpol.2013.02.022. 